\title{
Antoponimia plurilingue: storia della lingua e storia delle identità
}

un progetto di ricerca sull'onomastica personale e familiare in VDA

\section{Gianmario Raimondi}

\section{(2) OpenEdition}

\section{Journals}

Edizione digitale

URL: http://journals.openedition.org/esp/481

DOI: $10.4000 /$ esp.481

ISSN: 2532-0319

\section{Editore}

Centre d'Information sur l'Éducation Bilingue et Plurilingue

Edizione cartacea

Data di pubblicazione: 1 juin 2015

Paginazione: 15-24

ISSN: 1127-266X

Notizia bibliografica digitale

Gianmario Raimondi, «Antoponimia plurilingue: storia della lingua e storia delle identità», Éducation et sociétés plurilingues [Online], 38 | 2015, Messo online il 01 mars 2016, consultato il 25 février 2021. URL: http://journals.openedition.org/esp/481 ; DOI: https://doi.org/10.4000/esp.481 


\section{ANTROPONIMIA PLURILINGUE: STORIA DELLA LINGUA E STORIA DELLE IDENTITÀ: UN PROGETTO DI RICERCA SULL'ONOMASTICA PERSONALE E FAMILIARE IN VDA}

\section{Gianmario RAIMONDI}

Cette contribution montre la genèse, l'état actuel et les perspectives de développement futur d'un projet de recherche sur l'onomastique personnelle, financé par l'Université de la Vallée d'Aoste, avec la collaboration de l'Assessorat régional Education et Culture, Antroponimia Plurilingue (AntroPlur 2013-2015). Constitué à partir de la base de données existante de l'ASAVDA (Archives Historiques Anthroponymiques de la Vallée d'Aoste), ce projet, qui profite de la collaboration de chercheurs aussi bien locaux que d'autres universités italiennes, se propose d'intégrer le focus originel historique et linguistique des archives, en investiguant tout particulièrement, selon des perspectives interdisciplinaires, le rapport entre l'onomastique et l'" identité linguistique » dans un territoire comme celui de la Vallée d'Aoste, très intéressant à cause de sa configuration historique plurilingue.

This article details the genesis, state of the art and future development of a research project on personal onomastics financed by the University and the Department of Eduction of the Aosta Valley (Antroponimia Plurilingue - AntroPlur 2013-2015). Based on the ASAVdA data base (Archivio Storico Antroponimico della Valle d'Aosta), and with the collaboration of local as well as other as other Italian university research scholars, the project seeks to reintegrate the original historical and linguistic focus of the archives, investigating in particular the relationship between onomastics and "linguistic identity", in a region such as the historically plurilingual Aosta Valley.

Key words: Personal onomastics, Aosta Valley, linguistic identity, plurilingual configuration

Parole chiavi: Onomastica personale, Valle d'Aosta, identità linguistica, configurazione plurilingue

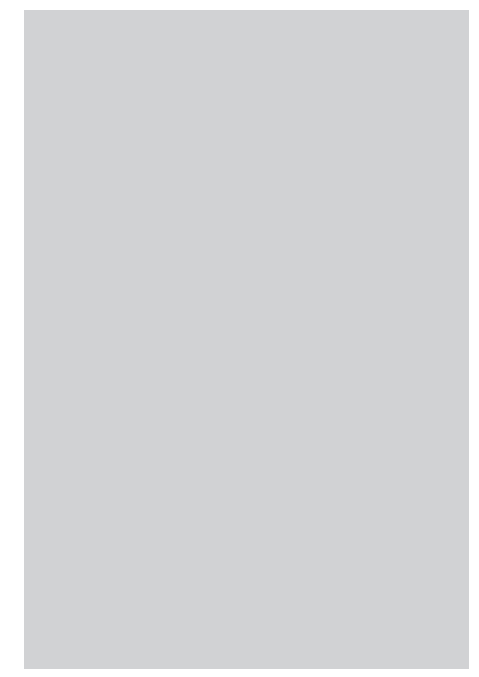

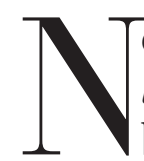

Tel quadro dei PRIA 2012-Progetti di Ricerca di vilevante Interesse per l'Ateneo, l'Università della Valle d'Aosta ha finanziato il progetto biennale di ricerca, da me coordinato, Antroponimia Plurilingue: storia della lingua e storia delle identità (AntroPlur), che ha preso avvio nel 2013 e sta avviandosi ora alla sua conclusione, prevista nel giugno 2015 per la sua fase operativa, cui seguiranno le attività di restituzione che si svilupperanno nel corso dell'anno.

Presentiamo qui le linee essenziali del progetto, relativamente alla sua storia e agli obiettivi specifici che lo caratterizzano, iscrivibili in linea generale in un territorio scientifico multidisciplinare al confine fra l'onomastica, la ricerca storica e storico-linguistico, la lessicografia e la dialettologia gallo- e italoromanza, la linguistica di contatto e la sociolinguistica. 
Antroponimia plurilingue: storia della lingua e storia delle identità in VDA

G. RAIMONDI

ASAVDA: UNA BASE DI DATI STORICOLINGUISTICA
Il progetto costituisce la continuazione di una delle linee di ricerca del suo coordinatore, iniziata nel 2001 a seguito di un finanziamento ministeriale italiano (CNR-Agenzia 2000), la quale nel corso degli anni ha condotto alla creazione di un data base (ASAVdA-Archivio Storico Antroponimico della Valle d'Aosta) che raccoglie e ordina una mole cospicua di documentazione antroponimica datata e georeferenziata riferentesi al territorio valdostano fra il XIV e il XVIII secolo.

In relazione ai presupposti metodologici, il progetto ASAVdA si è originato nel solco della tradizione degli studi antroponomastici europei degli anni Ottanta-Novanta del secolo trascorso ('esempio più illustre dei quali è rappresentato da PATROM-Patronymica Romanica) (1), volti allo studio comparato dell'antroponimia personale (nomi di battesimo) e familiare (cognomi) dei territori neolatini attraverso la creazione di ampi repertori di antroponimi tratti da documentazione archivistica che parte dall'Alto Medioevo fino alla contemporaneità, in chiave sia sincronica che diacronica.

Nello specifico, il progetto ASAVdA mirava alla costruzione di un analogo sistema di documentazione antroponimica quanto più esaustiva possibile, cercando però di muoversi nella dimensione più controllabile di un territorio regionale quale la Valle d'Aosta; un territorio caratterizzato anche da una peculiare abbondanza di documentazione storicoarchivistica, dovuta sia al ruolo di centro vescovile svolto da Aosta dalla fine della latinità ad oggi, sia alla natura "autarchica" delle dinamiche di potere interne (presenza di signorie locali autonome e poi costituzione in sede ducale), sia alla dipendenza da una casa regnante, quella dei Savoia, particolarmente attenta alla cura della documentazione amministrativa e fiscale, indispensabile per le finalità specifiche della ricerca storico-documentaria di interesse onomastico.

L'interesse della Valle d'Aosta, inoltre, discende anche dal suo costituire al tempo stesso un territorio delimitato e unitario dal punto di vista della caratterizzazione etno-linguistica (territorio di montagna, appartenente nel suo insieme alla sub-area francoprovenzale del dominio galloromanzo), ma anche un importante crocevia di dinamiche storicoeconomiche e politiche (si pensi al ruolo svolto in epoca antica e medievale dai passi del Grande e del Piccolo San Bernardo, che mettevano in comunicazione l'area rodaniana svizzera e francese con la pianura del Po e l'Italia) che lo arricchiscono di una variegata storia di plurilinguismo e di molteplici influssi culturali concorrenti (2).

Nel decennio 2001-2012, la banca dati si è così costituita attraverso lo spoglio e il caricamento informatico di nomi di persona tratti da una serie di fonti documentarie edite e inedite, che comprendono (cfr. Raimondi 2012: 129 e la bibliografia cui si rimanda): 1) Per i secoli XIV-XVI la documentazione fiscale inedita dei Comptes de 
Antroponimia plurilingue: storia della lingua e storia delle identità in VDA

G. RAIMONDI
ANTROPLUR: DALLA STORIA DELLA LINGUA ALL'IDENTITÀ
Subside/Conti di Sussidio conservata all'Archivio di Stato di Torino e all'Archivio Storico Regionale di Aosta (cfr. Frutaz 1966), e riferibile alle castellanie sabaude medievali di Aosta-Châtelargent-Valdigne, Quart-Oyace-Valpelline, Monjovet, Cly e Bard; 2) Per lo stesso periodo, una serie di materiali tratti da tesi di laurea dell'Università di Torino (realizzate sotto la supervisione di Giuliano Gasca Queirazza S.J., illustre e compianto studioso di onomastica) riferite alle località di Quart, Cogne e Montjovet; 3) Per i secoli XV-XVI, il repertorio onomastico estraibile dall'edizione Zanolli dei Computa Sancti Ursi e dei registri del Castello di Issogne; 4) Per il XVIII secolo, le liste dei proprietari censite dai Catasti Sardi per tutto il territorio valdostano e pubblicate da Berton fra il 1976 e il 1988.

L'approccio naturale di studio per un repertorio costituito come l'ASAVdA è ovviamente ed essenzialmente storico-linguistico e le sue ricadute in termini di conoscenza si riferiranno ai settori della dialettologia galloromanza, della storia della lingua e dell'etimologia. Il suo prodotto immediato può infatti essere rappresentato nella forma tradizionale di un "dizionario storico ed etimologico" dei nomi di persona e dei cognomi valdostani più o meno antichi; un repertorio, quindi, che presenti i lemmi (nomi e cognomi, adeguatamente tipologizzati) corredati delle forme e delle attestazioni antiche e commentati in senso storico ed etimologico, sulla base di modelli ampiamente collaudati nelle pubblicazioni onomastiche. Nel caso particolare della banca dati ASAVdA, i lemmi avrebbero la possibilità poi di essere corredati da cartogrammi relativi alle frequenze e alla distribuzione diacronica e diatopica, poiché ogni singolo dato dell'archivio risulta localizzato nel tempo (per anno e per secolo) e nello spazio (sulla base della ripartizione amministrativa attuale in Comuni).

Solidamente ancorato a questa base preesistente, il progetto presentato nel 2012 da un lato costituisce la prosecuzione del lavoro di implementazione della banca dati, dall'altro rappresenta una nuova focalizzazione degli interessi di ricerca su ambiti complementari a quello storicolinguistico. Se infatti un repertorio di grande rilevanza statistica, come quello che l'ASAVdA verrebbe a costituire attraverso la raccolta dei dati, rappresenta una base assolutamente straordinaria per interpretare l'evoluzione del sistema antroponimico di un'area (quella valdostana) in maniera capillare, è vero anche che la complessità della storia e della storia linguistica della Valle d'Aosta cui si è accennato sopra permette ulteriori letture che, a partire da questa base documentaria, ne sappiano valorizzare e attualizzare l'importanza sotto una specifica luce interdisciplinare individuata nel concetto di "identità": un tema cui la recente riflessione antropologica (cfr. fra gli altri e per l'Italia Fabietti 
Antroponimia plurilingue: storia della lingua e storia delle identità in VDA

G. RAIMONDI
1998) ha assegnato un posto rilevante nell'agenda della cultura contemporanea, mettendo in evidenza i forti legami che esso intrattiene con gli elementi di tensione fra "individuale" e "collettivo", fra "locale" e "globale" che segnano profondamente la società post-moderna.

L'identità sociale (l'insieme di valori "che ci rende uguali ad altri e al tempo stesso diversi da altri", come si potrebbe dire parafrasando la Social Identity Theory di Tajfel) si esprime anche attraverso la lingua. E nelle situazioni di "pidginizzazione" o, più genericamente, di "contatto" fra culture etnicamente diverse, o etnicamente contigue ma fra loro socialmente subordinate, la condizione intrinsecamente conflittuale che si genera può dar vita a fenomeni reattivi, quali lo "stigma sociale" o all'opposto la "lealtà linguistica" (secondo le dinamiche oppositive ben descritte già da Weinreich 1974: 144); oppure, viceversa, a fenomeni di appiattimento delle differenze che generano il conflitto, quale l'adozione integrale, come modello linguistico, della "lingua tetto", la lingua cioè che viene assunta come codice privilegiato per le funzioni "alte" e ufficiali della comunicazione (Kloss 1976).

Per la Valle d'Aosta (terra di "frontiera" e quindi di contatto linguistico per eccellenza), sia la dimensione storica che quella contemporanea mostrano che le diverse fasi di conflitto fra culture linguistiche dominanti, e fra lingue dominanti e lingue subalterne, hanno determinato una fenomenologia che è attestazione di entrambe le tendenze illustrate; e che questo vale in linea di principio anche per i fenomeni osservabili nella storia dei nomi di persona, che mostrano, nell'evoluzione della loro forma, sia la spinta verso l'adattamento che la tendenza opposta del mantenimento dei loro caratteri linguistici originali e dialettali (3).

Su queste premesse si è strutturato il nuovo progetto, il quale si è proposto di far ruotare intorno all'oggetto "ASAVdA" una serie di azioni di ricerca correlate volte al contempo: a) a valorizzarne l'originale dimensione storico-linguistica, lessicografica e specificamente onomastica; b) ad integrare con nuovi sondaggi, sui periodi storici e sulla tipologia di documentazione non ancora utilizzata nelle fasi precedenti, e anche nella contemporaneità, la rappresentatività statistica della banca dati; c) ad ampliare il suo utilizzo in una nuova dimensione aperta verso tematiche interdisciplinari di carattere sia teoretico che applicativo, nei campi della linguistica (in particolare secondo prospettive linguistiche di carattere dialettologico italo- e galloromanzo, sociolinguistico, etnolinguistico e filosofico-teoretico) e dello studio storico-archivistico (fonti per la storia, diplomatica), al fine di fornire una lettura quanto più ricca possibile della storia identitaria valdostana attraverso lo spaccato 
Antroponimia plurilingue: storia della lingua e storia delle identità in VDA

G. RAIMONDI dell'onomastica personale. A supporto di queste azioni di ricerca, il progetto prevedeva inoltre l'attivazione di una consulenza informatica volta all'implementazione della struttura relazionale dell'archivio esistente (procedure automatizzate di lemmatizzazione e di classificazione), allo sviluppo di un'interfaccia per gli output editoriali previsti (vedi sotto) e alla realizzazione del sito nelle due modalità di amministrazione in remoto delle operazioni di caricamento e ordinamento e di gestione dei contenuti lato-utente (consultabilità on line di materiali selezionati e possibilità di interrogazione on line delle parti autorizzate dell'archivio).

Ai fini scientifici descritti sopra, sono stati coinvolti nel progetto ricercatori di diversa provenienza e di diversa estrazione disciplinare, in particolare: Luisa Revelli (storica della lingua italiana e studiosa di onomastica), Laura Balbiani (germanista) e Giuseppe Landolfi Petrone (filosofo del linguaggio) per l'Università della Valle d'Aosta; Alessandro Vitale Brovarone (romanista), Tullio Telmon (dialettologo) e Sabina Canobbio (dialettologa ed etnolinguista) per l'Università di Torino; Rita Caprini (glottologa e specialista di onomastica) per l'Università di Genova. Nell'ambito locale, al progetto hanno dato inoltre il loro supporto l'Archivio Storico Regionale di Aosta, nella persona di Roberto Bertolin (archivista e cultore di storia locale), e il Bureau Régional pour l'Ethnographie et la Linguistique, attraverso la partecipazione di Saverio Favre (francoprovenzalista e specialista di cultura locale).

A dimostrazione dell'interesse che il progetto riveste a livello di ricaduta sulla comunità e sul territorio, l'iniziativa ha ricevuto la disponibilità al cofinanziamento dell'Assessorato Istruzione e Cultura della Regione Autonoma Valle d'Aosta, che ha messo a disposizione il cofinanziamento al 50\% di un assegno di ricerca, e della Banca di Credito Cooperativo Valdostano, la quale si è assunta l'onere del finanziamento delle pubblicazioni (4). I prodotti della ricerca attesi consistono in una doppia pubblicazione: 1) Il DSEAV-Dizionario Storico-Etimologico dell'Antroponimia Valdostana, che raccoglierà le attestazioni tratte dalla documentazione ASAVdA in lemmi commentati storicamente ed etimologicamente in due sezioni, riferite ai nomi personali (o "di battesimo") e di famiglia (o "cognomi"); 2) Un volume di saggi di approfondimento, che ospiterà i contributi di tutti i partecipanti al progetto, invitati a "leggere" secondo le rispettive competenze il rapporto fra onomastica personale, identità linguistica e identità culturale, alla luce dei dati contenuti nell'archivio oppure sulla base di collegamenti di questi con temi anche generali ma pertinenti, riferibili ai campi propri dell'onomastica (Raimondi, Revelli, Caprini), della dialettologia franco- 
Antroponimia plurilingue: storia della lingua e storia delle identità in VDA

G. RAIMONDI
ANTROPLUR: STATO DELL'ARTE $(31.01 .2015)$ provenzale (Telmon, Favre), della linguistica galloromanza (Vitale Brovarone), della metodologia lessicografica (Raimondi, Balbiani), delle teorie del nome proprio (Raimondi, Landolfi), della sociolinguistica (Raimondi, Revelli), dell'etnolinguistica (Canobbio, Caprini), della diplomatica (Raimondi, Bertolin). In prospettiva di disseminazione dei contenuti del progetto, il programma prevede anche 3) la pubblicazione definitiva del sito rererreasavda.it, ad oggi attivo solo in modalità backoffice, che risulterà anche funzionale all'implementazione futura dell'archivio, accessibile anche in remoto per il caricamento dei dati attraverso procedure controllate dall'amministratore. L'interesse dimostrato da quanti (storici locali ed appassionati) operano da anni singolarmente nel campo dell'onomastica attraverso la ricerca negli archivi locali, comunali e parrocchiali, potrà infatti concretizzarsi nella forma della contribuzione libera attraverso l'integrazione dei loro materiali documentari nella banca dati.

A seguito del finanziamento ottenuto, oltre all'assegno di ricerca previsto (titolare Claudia Alessandri, già collaboratrice redazionale dell'ATPM-Atlante Toponomastico del Piemonte Montano) e alla consulenza informatica (Andrea Guarda), è stato attivato un contratto di collaborazione (titolare Luca Jaccod, archivista presso l'Archivio Diocesano di Aosta) che ha permesso di procedere con l'integrazione nella banca dati di un campione di documentazione tratto da una fonte finora non utilizzata, i registri battesimali parrocchiali.

Il campionamento effettuato sul secolo XVII, fino ad ora scoperto, ha condotto a selezionare 12 località distribuite uniformemente sul territorio regionale (Aosta, con le due parrocchie di Saint-Jean e Saint-Laurent, Saint-Oyen, Châtillon, Issime importante in quanto sede di una delle comunità walser valdostane - e Gaby, Cogne, Brusson, Arnad, Champorcher, AnteySaint-André, registrato insieme a Chamois e La-Magdeleine, Pont-Saint-Martin, Morgex e Saint-Christophe) che sono in corso di caricamento sul data base (circa 5.000 individui). Allo stato attuale (mentre la revisione dell'archivio è ancora in corso), il data base ASAVdA conta 66.271 individui censiti, che determinano circa 120.000 registrazioni di forme nominali (fra nomi di battesimo e cognomi). Le registrazioni degli individui mostrano la seguente distribuzione per secolo: sec. XIV, $5.926(9,0 \%)$; sec. XV, 10.065 (15,2\%); sec. XVI, 2.275 (3,4\%); sec. XVII, 7.580 $(11,4 \%)$; sec. XVIII, 46.351 (69,9\%). Il taglio sincronico più rilevante è quindi costituito dai dati settecenteschi dei Catasti Sardi, che del resto si riferiscono alla situazione onomastica precedente 
Antroponimia plurilingue: storia della lingua e storia delle identità in VDA

G. RAIMONDI alla mobilità territoriale che caratterizza il secolo successivo e possono quindi essere considerati rappresentativi (dal punto di vista distribuzionale diatopico) del panorama tradizionale di epoca "moderna" (non contemporanea), e sono inoltre completi per quanto riguarda la copertura territoriale.

La Tabella 1 mostra la distribuzione degli individui censiti nei 72 comuni valdostani indicizzati nella banca dati (5). Le proporzioni statistiche sono rilevanti: per il capoluogo Aosta, i 6.378 individui censiti rappresentano ad esempio circa il 12\% della popolazione attuale (35.031, al censimento del 2011) e la percentuale sale per alcuni centri minori ben rappresentati come Châtillon, dove sfiora il $30 \%$ (1.626 contro 4.966). Il numero di registrazioni dipende anche dalla documentazione disponibile, dato che quella più antica (i Conti di Sussidio) si riferisce solo alle castellanie amministrate direttamente dai Savoia (ben coperte sono ad esempio tutta la Valdigne e la Valle del Gran San Bernardo), mentre per gli spogli dei registri battesimali si è dovuto fare i conti con la loro conservazione e leggibilità effettive e con il tempo necessario al lungo lavoro di trascrizione, che ha orientato a limitare, per ora, l'accesso a queste fonti dal punto di vista quantitativo. In relazione a questo, nella tabella lo sfondo bianco identifica le località per le quali è disponibile per ora solo la documentazione settecentesca dei Catasti; quello grigio medio i comuni per cui è presente almeno un'altra fonte più antica; il fondo scuro contrassegna invece le località selezionate per una copertura diacronica integrale: ovviamente Aosta, una località di dimensioni notevoli dell'Alta Valle (Morgex), due più piccole per la Bassa Valle (Champorcher, cui si aggiungerà Pont-Saint-Martin, qui segnata come non ancora completata), Saint-Christophe come rappresentativa dei centri più grandi della Plaine a est del capoluogo, Cogne come località periferica e ugualmente rappresentativa.

Nella fase attuale è inoltre allo studio la possibilità di acquisire e riversare direttamente nel data base l'archivio anagrafico del Comune di Aosta, operazione che consentirebbe un ulteriore taglio sincronico sulla contemporaneità. La possibilità di accedere a dati statistici sulla contemporaneità consentirebbe di affrontare, fra le possibili letture della banca dati, altri due argomenti particolarmente sensibili per rapporto alla nozione di "identità" collegata all'onomastica che il progetto intende approfondire. 


\begin{tabular}{|c|c|c|c|c|c|}
\hline COMUNE & $\begin{array}{l}\text { NR. } \\
\text { IND. }\end{array}$ & COMUNE & $\begin{array}{l}\text { NR. } \\
\text { IND. }\end{array}$ & COMUNE & $\begin{array}{l}\text { NR. } \\
\text { IND. }\end{array}$ \\
\hline Allcin & 252 & Émarèse & 302 & Pontey & 302 \\
\hline Antey-Saint-André & 1104 & Etroubles & 798 & Pont-Saint-Martin & 440 \\
\hline Aosta & 6378 & Fénis & 843 & Pré-Saint-Didier & 713 \\
\hline Arnad & 621 & Fontaincmore & 549 & Quart & 2453 \\
\hline Arvier & 690 & Gignod & 1520 & Rhèmes-Notre-Dame & 179 \\
\hline Avise & 386 & Gressan & 731 & Rhemes-Saint-Georges & 276 \\
\hline Ayas & 704 & Gressoney la Trinité & 483 & Roisan & 509 \\
\hline Aymavilles & 725 & Gressoney-Saint-Jean & 195 & Saint-Christophe & 1976 \\
\hline Bard & 335 & Höne & 429 & Saint-Denis & 519 \\
\hline Bionaz & 206 & Introd & 351 & Saint-Marcel & 479 \\
\hline Brissogne & 564 & Issime & 1091 & Saint-Nicolas & 281 \\
\hline Brusson & 1214 & Issogne & 326 & Saint-Oyen & 405 \\
\hline Ghalland-Saint-Anselme & 320 & Jovençan & 368 & Saint-Pierre & 774 \\
\hline Challand-Saint-Victor & 695 & Lat Salle & 2409 & Saint-Rhémy-en-Bosses & 855 \\
\hline Chambave & 447 & Ia Thuile & 636 & Saint-Vincent & 1478 \\
\hline Chamois & 150 & Lilianes & 419 & Sarre & 1266 \\
\hline Champdepraz & 252 & Montjovet & 1413 & Torgnon & 524 \\
\hline Champorcher & 1067 & Morgex & 2087 & Valgrisenche & 188 \\
\hline Charvensod & 323 & Nus & 1586 & Valpelline & 380 \\
\hline Châtillon & 1626 & Ollomont & 426 & Valsavarenche & 578 \\
\hline Cogne & 2153 & Oyace & 321 & Valtournenche & 513 \\
\hline Courmayeur & 1337 & Pertoz & 623 & Vertayes & 798 \\
\hline Donnats & 1341 & Pollein & 317 & Verres & 747 \\
\hline Doues & 524 & Pontboset & 345 & Villeneuve & 104.1 \\
\hline
\end{tabular}

Tabella 1: Copertura della banca dati ASVdA per comune (al 31.01.2015)

Da un lato, diverrebbe possibile sondare le tendenze più recenti in relazione alle scelte onomastiche battesimali dei valdostani, nel rapporto fra una tradizione onomastica fortemente legata alle devozioni locali, che appare ormai un ricordo del passato (6), una tradizione "italiana" impostasi progressivamente nell'ultimo secolo e mode onomastiche più recenti, nelle quali il gusto per l'esotismo che contrassegna in parte l'onomastica nazionale di oggidì pare sposarsi in Valle con l'accostamento all'onomastica francofona, con significato evidentemente identitario.

Da un secondo punto di vista, i dati contemporanei potrebbero comple- 
Antroponimia plurilingue: storia della lingua e storia delle identità in VDA

G. RAIMONDI

\section{BIBLIOGRAFIA}

tare il quadro dell'identità valdostana con le evidenze della sua configurazione più recente, nella quale la presenza di cognomi "allogeni", soprattutto generati dalla migrazione interna del Secondo Dopoguerra, è imponente, poiché nei primi 10 ranghi dell'onomastica familiare del capoluogo si trovano ben otto cognomi di origine calabrese (reggina, in particolare): 1) Mammoliti, 2) Fazari, 3) Giovinazzo, 4) Raso, 8) Romeo, 9) Agostino, 10) Tripodi.

In che misura questo sovvertimento del panorama onomastico familiare incide sulla percezione dell'identità locale da parte dei valdôtains de souche? Fino a che punto l'integrazione, apparentemente priva di problemi e profonda, di questi "nuovi" valdostani è capace di incidere sulle configurazioni identitarie complessive del tessuto sociale valdostano? I risultati della riflessione condotta lungo il filo del progetto Antroponimia plumilingue potranno, nei nostri auspici, portare un contributo anche a questioni socialmente rilevanti come queste.

CANO GONZALES A. M., GERMAINJ., KREMER D. (eds.). 2004. Dictionnaire historique de l'anthroponymie romane (PatRom). Vol. II.1: L'homme et les parties du corps humain, Tübingen: Niemeyer.

FABIETTI U. 1998. Lidentità etnica. Storia e critica di un concetto equivoco, Roma: Carocci ( $2^{\text {nd }}$ ed; $1^{\text {st }}$ ed. 1995, Roma: La Nuova Italia Scientifica).

FAVRE S. 2002. La Valle d'Aosta, pp. 139-150 in M. CORTELAZZO et al. (eds.), I dialetti italiani. Storia, struttura, uso, Torino, UTET.

FRUTAZ A. P. 1966. Le fonti per la storia della Valle d'Aosta, Aosta: Tipografia Valdostana.

KLOSS H. 1976. Abstandsprachen und Ausbausprachen, pp. 301-322 in J. GÖSCHEL, N. NAIL, G. VAN DER ELST (eds.), Zur Theorie des Dialekts, Wiesbaden: Franz Steiner Verlag («Zeitscrift für Dialektologie und Linguistik» 16).

RAIMONDI G. 2006. Storia e configurazione del repertorio plurilingue valdostano, pp. 100-126 in F. BERTOLINO, L. REVELLI (eds.), Università, scuola, territorio. Percorsi integrati per la formazione dell'insegnante promotore delle risorse del territorio, Milano: Franco Angeli.

RAIMONDI G. 2012. I nomi di persona nella Valle d'Aosta fra XIV e XVIII secolo. Interferenze linguistiche, interferenze culturali, Aosta: Le Château.

REVELLI L. 2010. Cognomi come nomi, nomi come cognomi. Appellativi fermminili in Piemonte e Valle d'Aosta, pp. 385-394 in M. ILIESCU, H. SILLERRUNGGALDIER, P. DANLER (eds), Actes du XXVe Congrès International de Linguistique et de Philologie Romanes (Innsbruck 2007), Vol. 3, Berlin/New York, De Gruyter. 
Antroponimia plurilingue: storia della lingua e storia delle identità in VDA

G. RAIMONDI
WEINREICH U.1974 [1953]. Lingue in contatto, con saggi di Francescato, Grassi e Heilmann, Torino: Boringhieri $\left(1^{\circ}\right.$ ed.: Languages in contact. Findings and Problems, New York, Linguistic Circle of New York).

\section{NOTE}

(1) Il progetto internazionale, coordinato inizialmente dall'Università di Treviri, ha prodotto in questi anni una serie cospicua di pubblicazioni specialistiche (per i tipi di Niemeyer e De Gruyter), fra cui monografie su specifici contesti storico-geografici, approfondimenti metodologici e il primo volume del Dictionnaire historique de l'anthroponymie romane (Cano Gonzales/Germain/Kremer 2004).

(2) Per un profilo storico-linguistico riassuntivo si vedano Favre $2002 \mathrm{e}$, in particolare per la delineazione delle fasi diglottiche più antiche, Raimondi 2006. Fra le lingue e le culture in gioco, non si dovrà dimenticare che la valdostana Valle del Lys ospita anche un'altra importante minoranza linguistica storica, quella alemannica dei Walser.

(3) Le fasi conflittuali ai livelli "alti" del repertorio sono quella che oppone il latino e il francese nel volgere del Medioevo verso l'Età Moderna e poi quella che, dopo l'Unità d'Italia, al francese oppone l'italiano, prima "lingua nazionale unitaria" a fare il suo ingresso nelle dinamiche sociolinguistiche della regione. Ai gradini inferiori, la dialettica sociolinguistica vede innanzitutto le varietà francoprovenzali locali, che non hanno mai mostrato la propensione alla costruzione di una varietà sovralocale di riferimento e sono quindi rimaste sempre nello stato di "dialettalità pura"; accanto ad esse, in posizione dapprima (XVII-XIX secolo) intermedia nel repertorio, poi recessiva, il piemontese di koinè impostosi gradualmente nei centri del Fondovalle e poi soppiantato dall'italiano (cfr. Raimondi 2006). Per le dinamiche specifiche dell'adattamento diglottico nel campo dell'onomastica personale, cfr. in particolare Revelli 2010 e Raimondi 2012: 29-46.

(4) Il successo dell'iniziativa è stato garantito anche da una serie di convenzioni che l'Ateneo valdostano aveva già stipulato con alcuni dei soggetti promotori, in particolare quella con l'Assessorato all'Istruzione e cultura della Regione Valle d'Aosta (2006-2008-2010), della quale il progetto ASAVdA faceva già parte, e quella con il Dottorato di Ricerca in Dialettologia, geolinguistica e sociolinguistica (2012), cui aderiscono 5 componenti del gruppo di ricerca. Citiamo infine il supporto morale fornito anche dalla convenzione con il CIEBP-Centre International pour l'Éducation Bilingue e et Plurilingue, che al progetto ha collaborato esternamente.

(5) Mancano i comuni di Gaby (censito sempre con Issime) e La Magdeleine (con Antey-Saint-André), che sono di istituzione recente. Si tenga presente, inoltre, che in diversi casi le attribuzioni territoriali antiche delle frazioni (basate sulle pertinenze parrocchiali) non corrispondono a quelle amministrative attuali.

(6) Cassiano (martire del III secolo, patrono di La Salle), Pantaleone (medico e martire a Nicomedia, venerato nelle sue spoglie a Lione e in tutta la Francia orientale), Giocondo (secondo vescovo di Aosta) sono ad esempio alcuni dei nomi tradizionali valdostani più insoliti, tutti legati a santi particolarmente venerati nella diocesi aostana. Per l'onomastica devozionale valdostana, cfr. Raimondi 2012: 82-103. 